\title{
Global and local vision in natural scene identification
}

\author{
Andrea De Cesarei • Geoffrey R. Loftus
}

Published online: 21 July 2011

(C) Psychonomic Society, Inc. 2011

\begin{abstract}
The results of previous studies have suggested that to optimize the decoding of visual information, global contents of a scene are analyzed before local features (global precedence hypothesis). Evidence supporting this hypothesis has been provided for identification of characters, faces, hybrid stimuli, and simple objects. In the present study, we examined identification of highand low-pass filtered natural pictures. Despite the radical differences in the type of information conveyed by global and local features, confident and accurate identification was achieved on the basis of either kind of information when an intermediate range of spatial frequencies was preserved. The present data are consistent with the notion of global precedence in scene identification.
\end{abstract}

Keywords Perceptual categorization and identification . Global precedence $\cdot$ Spatial frequencies $\cdot$ Visual perception

Humans can accurately identify visual scenes, even when incoming visual information is degraded. For instance, fewer fine-grained details are perceivable in a distant as compared with a near object, or when the contrast is lowfor example, when it is foggy. Nevertheless, some objects can be identified even with degraded visual conditions. Which kinds of information are critical for scene identifi-

\section{A. De Cesarei $(\square)$}

Department of Psychology, University of Bologna,

Viale Berti Pichat, 5,

40127 Bologna, BO, Italy

e-mail: andrea.decesarei@unibo.it

\section{G. R. Loftus}

Department of Psychology, University of Washington,

Box 351525, Seattle, WA 98195-1525, USA

e-mail: gloftus@u.washington.edu cation? In the present study, we examined the role of global and local information in the identification of natural scenes.

It has been proposed that in order to achieve efficient object identification, the human visual system analyzes the contents of visual scenes in a temporal order defined by the spatial characteristics of its elements (Navon, 1977). More specifically, the richness of details that populate a visual scene can be organized by spatial scale, which ranges continuously from "global" to "local" properties (see Fig. 1). Global features of a scene describe coarse characteristics such as the division between the sky and the landscape, whereas local features involve brightness changes across a small amount of space and convey information about fine-grained details such as spots on a cheetah's back. Global and local characteristics can also be described as changes in an image's spatial frequenciesthat is, in the number of times one detail can be repeated in a unit of space (Shulman \& Wilson, 1987). In particular, low and high spatial frequencies describe respectively elements that cover large areas of an image (low spatial frequencies or global features) or small portions of a scene (high spatial frequencies or local features). It has been suggested that in order to optimize efficiency in scene recognition, a sequence of stages analyzes progressively smaller portions of the visual field, resulting in earlier identification of global than of local stimulus features (Bar, 2004; Navon, 1977).

Evidence supporting the hypothesis that processing of global elements precede processing of local details has been obtained in a number of studies investigating identification of digits, numbers, line drawings, and faces (Loftus \& Harley, 2004; Navon, 1977; Sanocki, 1993). Typically in these studies, a first stimulus that is either global or local is presented for a variable exposure time, followed by a test stimulus that has to be matched to the first stimulus. These 

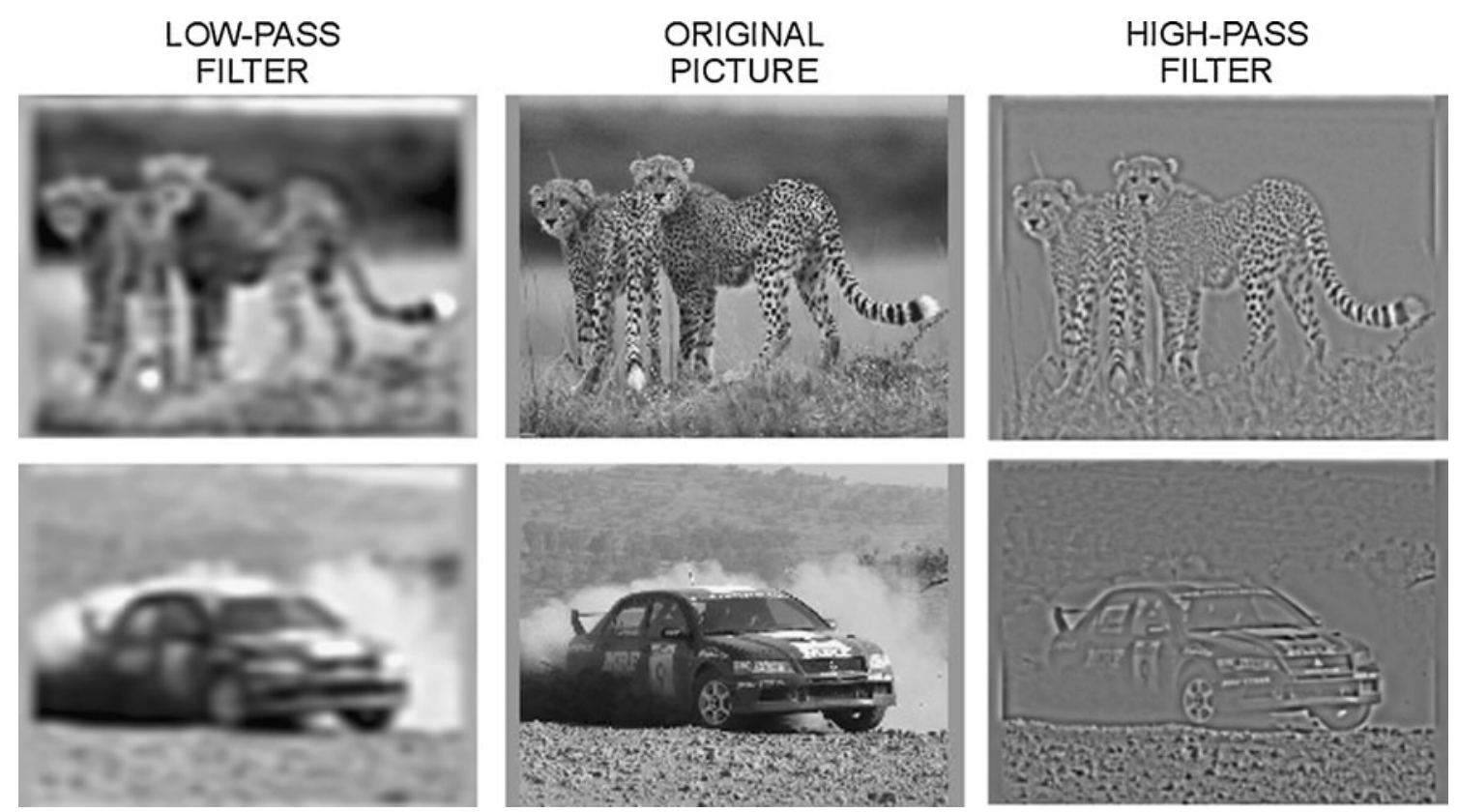

Fig. 1 Original, low-passed, and high-passed version of two sample pictures. Associated descriptors (tasks DC-YN and DC-C) were cheetas (upper) and a car (bottom)

studies demonstrate an advantage for global as compared with local stimuli at short exposure times, and this advantage is interpreted as reflecting a processing sequence whereby global processing precedes local processing (Loftus \& Harley, 2004). In addition, some studies have investigated the identification of hybrid images, which are composed of a low-spatial-frequency version of one picture (e.g., a city landscape) superimposed on a high-spatial-frequency version of a different picture (e.g., a close-up view of a house). When such hybrid pictures are shown for short durations, the lowspatial-frequency picture is usually perceived, whereas with longer durations, the high-spatial-frequency picture is usually perceived (Schyns \& Oliva, 1994). Finally, psychophysiological evidence has suggested a feedback model of visual analysis, where early global information biases subsequent finer scale processing (Bar, 2004; Bullier, 2001; Fabre-Thorpe, 2003). In this model, a scene is initially analyzed to extract global information, and the result of this raw analysis is projected to brain structures which bias further fine-scale fine-scale processing based on task relevance or emotional value of the scene (Bar, 2004).

The present study was aimed at assessing the identification of natural pictures by their global or local properties. Previous work has reported evidence supporting the global precedence hypothesis in the identification of impoverished, simple, or artificial stimuli (line drawings, digits, hybrid images; Loftus \& Harley, 2004; Navon, 1977; Sanocki, 1993; Schyns \& Oliva, 1994). A straightforward prediction of the global precedence hypothesis is that even when viewing highly complex stimuli such as natural scenes, a temporal advantage for global as compared with local features will be observed. To this end, low- and high-passed versions of natural scenes were presented at an either short or long exposure time, and picture identification was assessed.

\section{Method}

For clarity, we will briefly describe the general design and the tasks used in this study before going into their details.

\section{Task and design synopsis}

On each of a series of trials, a naturalistic scene was presented in one of - depending on the task-either 12 or 16 conditions defined by either three or four levels of desired performance level, along with two levels of filter type (high pass vs. low pass), and two levels of picture exposure duration $(0.1 \mathrm{~s}$ or $1 \mathrm{~s})$. Desired performance levels were achieved by adaptively varying the filter's strengththat is, the range of spatial frequencies eliminated by the filter.

The study was aimed at investigating the role of spatial frequencies in gist understanding. To this end, three tasks were designed that differed only in how gist understanding was assessed. We used three different tasks for generalitythat is, to limit the possibility that results were related to specific features of a task such as the specific verbal 
descriptor, or the decision processes. The three tasks are as follows:

DC-YN ("Description correct-yes or no"). A naturalistic scene was shown to a participant along with a brief description. The participant's task was to judge, with a yes-no response, whether the description matched the scene's gist.

DC-C ("Description correct-confidence"). A naturalistic scene was shown to a participant, along with a brief description. The participant's task was to provide a confidence rating of whether the description matched the scene's gist.

UG-YN ("Understand gist-yes or no"). A naturalistic scene was shown to a participant. The participant's task was to judge, with a yes-no response, whether he or she understood the scene's gist.

\section{Participants}

A total of 120 students (66 females, 54 males, mean age $=19.1, S D=1.54$ ) from the University of Washington, participating for course credit, were randomly assigned to one of the three task conditions. Because of the failure of the data acquisition algorithm, data in some blocks were not estimated. Participants who had missing data were excluded from analysis. Final $n \mathrm{~s}$ were: $\mathrm{DC}-\mathrm{YN}=28$; $\mathrm{DC}-\mathrm{C}=52$; $\mathrm{UG}-\mathrm{YN}=40$

\section{Stimuli and equipment}

A total of 350 grayscale pictures representing objects, people, urban, or naturalistic scenes were selected. For each picture, a verbal descriptor was created (descriptor length $M=1.92$ words, $S D=1.1$ ). Visual angle subtended by pictures was $21.07^{\circ}$ horizontal $\times 15.62^{\circ}$ vertical. Pictures were presented on eMac computers using the Psychophysics Toolbox (Pelli \& Zhang, 1991). Examples of two of the stimuli, along with gist descriptors, are provided in Fig. 1.

\section{Filter parameters}

The low-pass filter has been described in previous work (De Cesarei \& Codispoti, 2008, 2010; Loftus \& Harley, 2005): Its value was 1.0 (spatial frequencies were passed) up to some image spatial-frequency value $\mathrm{F}_{0}$ cycles/image, then declined parabolically with $\log$ spatial frequency, reaching zero at spatial frequency $\mathrm{F}_{1}=3 \cdot \mathrm{F}_{0}$, and remaining at zero for all spatial frequencies greater than $F_{1}$. The highpass filter was identical to the low-pass filter, mirror-imaged on the frequency axis; thus, it was zero up to $F_{1}$, then rose parabolically and asymptoted at 1.0 at $3 \cdot \mathrm{F}_{1}$. A graphical description of the filter can be found in a previous study
(Loftus \& Harley, 2005). Through the remainder of the article, a filter will be described by its direction (low pass or high pass) and its filter strength, defined as its cutoff value, $F_{1}$. Note that "greater filter strength" implies larger cutoff values for low-pass stimuli and smaller cutoff values for high-pass stimuli.

\section{Procedure}

A staircase procedure was applied (Levitt, 1971), which allows one to estimate the magnitude of some perceptual parameter-in our case, filtering strength, implemented in terms of filter cutoff - that leads to some prespecified performance level. This is done by adaptively varying filtering strength over trials according to a set of probabilistic rules. In general, the procedure is biased toward lower filter strengths for better identification accuracy, and higher filter strengths for poorer identification accuracy.

Separate blocks were run for each accuracy level, exposure duration, and filter type. Block order was randomized. Each block started from an initial cutoff value of 50.2 cpi (low-passed pictures) or 99.7 cpi (high-passed pictures), and initial step value was 2 octaves. ${ }^{1}$ On each trial within a block, an image was online filtered using either high-pass or high-pass filter with the appropriate filter cutoff $\left(\mathrm{F}_{1}\right)$ value, and presented for either $0.1 \mathrm{~s}$ or $1 \mathrm{~s}$. After each picture, identification accuracy was assessed as described below. Depending on identification after each trial, filter magnitude was increased, decreased, or left unchanged. On the next trial, a different picture was presented, and no picture was presented more than once. Whenever the filter magnitude changed direction (i.e., increased after having decreased, or vice-versa), step size was halved. After six direction changes, the procedure terminated, and the next block was started. On average, each block lasted 61.23 (DC-YN), 51.75 (DC-C), or 44.56 (UG-YN) pictures.

Estimation of filter magnitudes in the three tasks

In the DC-YN task, a descriptor was presented after each picture, and participants responded whether the description was correct or not. Participants were allowed to guess whether the picture and the descriptor matched, and 50\% accuracy was defined as "no memory." The psychophysical procedure was set to estimate filter strengths that implied accuracies of $70 \%, 80 \%$, and $85 \%$.

In the DC-C task, participants rated their confidence that the picture matched the descriptor on a 1 to 5 scale, where the confidence responses were defined as follows: $1=$

\footnotetext{
${ }^{1}$ All manipulations of spatial frequency to be described were done on a $\log _{2}$ cpi scale. We characterize frequencies as cpi for clarity.
} 
Correct, 2 = Probably correct, $3=I$ don't know, $4=$ Probably wrong and $5=$ Wrong. In the adaptive procedure, (Probably) correct and (Probably) wrong responses were treated as correct for match and mismatch trials, respectively. I don't know responses were treated as incorrect. These scoring conventions were chosen to ensure that participants' responses were not influenced by guessing when they did not know how to answer the question. Accordingly, 0\% accuracy corresponded to "no memory." With "accuracy" thus defined, the psychophysical procedure was set to estimate filter strengths that implied accuracies of $15 \%, 30 \%, 50 \%$, and $85 \%$.

Finally, in the UG-YN task, participants were asked whether they were able to understand picture content or not by pressing one of two keys. As with DC-C, $0 \%$ accuracy indicated failure to identify scene contents. The psychophysical procedure was set to estimate filter strengths that implied accuracies of $15 \%, 30 \%, 50 \%$, and $85 \%$.

\section{Data analysis}

As was suggested by Levitt (1971), initial values to the first two changes in direction were excluded to increase estimation efficiency. The resulting cutoff value was then defined as the mean of the remaining values. All resulting cutoff values greater than two standard deviations from the session mean were marked as outliers and discarded from analysis.

Our analysis relied mainly on the fitting of Weibull cumulative functions relating identification accuracy to filter cutoff. We assumed that that no identification resulted from filtering out of the whole picture, and $100 \%$ accuracy resulted from leaving the picture intact. The Weibull fits provided threshold and slope estimates (Pelli, 1987). Specifically, the threshold value indicates cutoff values that imply $80 \%$ (DC-YN) or 55\% (DC-C and UG-YN) identification accuracy. The slope parameter differentiates steep functions - in which identification accuracy varies from no identification to perfect identification over a small frequency range-from shallow functions, in which identification accuracy varies from no identification to perfect identification over a larger frequency range. To increase the signal-to-noise ratio in function fitting, and to be able to calculate confidence intervals around the estimated function values, a jackknife approach was used. Values for each individual participant were replaced by the average value of the remaining $n-1$ participants. Variances in each condition were then multiplied by $(n-1)^{2}$ to correct for the artificially small variances (Ulrich \& Miller, 2001).

\section{Results}

Figure 2 shows the fitted psychometric functions for all tasks, performance levels, and exposure durations, averaged across all participants. Note that when a low-pass spatial filter is applied (left panels), all spatial-frequency information exceeding the cutoff value is lost, leaving only lower spatial frequencies available for scene recognition. As the cutoff value is increased, more of the original picture content is preserved, and accuracy in scene identification increases. The opposite effect occurs for high-pass filtering (right panels): All frequencies below the filter cutoff are lost, and recognition becomes more difficult as the cutoff is shifted upward. A common feature of all tasks and conditions was the log-frequency symmetry of the psychometric functions estimated for high- and low-passed filtered stimuli: In particular, the average slope for low-passed pictures was $1.69(S E M=0.24)$ and for high-passed pictures it was $1.70(S E M=0.3)$.

In all tasks, the psychometric function shifted, with greater duration, to the more extreme portion of the frequency spectrum - that is, toward low spatial frequencies for low-pass filters, and toward high spatial frequencies for high-pass filters. In all task conditions, this effect was more pronounced for the high-passed than for the low-passed pictures, as is shown in Fig. 3 and in Table 1.

In DC-C and UG-YN, shallower slopes were observed when pictures were briefly presented, as compared with the longer exposure duration condition. Moreover, although in the brief exposure duration condition lower spatial frequencies exhibited a steeper slope than did higher spatial frequencies, the opposite was observed in the longer exposure duration condition (Table 2). Although only a trend in this direction was observed in DC-C, this effect was particularly pronounced in UG-YN. No changes in the slope of the psychometric function were observed in DC-YN.

\section{Discussion}

In three different tasks, high- and low-pass filter strengths were identified, which led to similar scene identification. Despite the radical differences in the type of information conveyed by high and low spatial frequencies, participants could nevertheless make efficient use of the available perceptual data to achieve semantically correct and confident representations of natural scenes.

\section{Global precedence for natural images}

The present study allowed a test of whether the use of lower and higher spatial frequencies varied with exposure duration. It did. In all tasks, the effects of exposure duration on identification accuracy were more pronounced for highthan for low-passed pictures, as indicated by the threshold shift of the psychometric functions. These results indicate 
Fig. 2 Cutoff $\left(\mathrm{F}_{1}\right)$ values and error bars for low-passed pictures (left panels) and highpassed pictures (right panels) for the three conditions. a description correct-yes or no; $\mathbf{b}$ description correct-confidence; c understand gist-yes or no. Dashed lines through the data points are best-fitting Weibull functions. Cutoff values are shown both in $\log _{2}$ CPI (upper) scales and linear CPI (bottom) scales
A

DC-YN (Description correct-yes or no) Condition
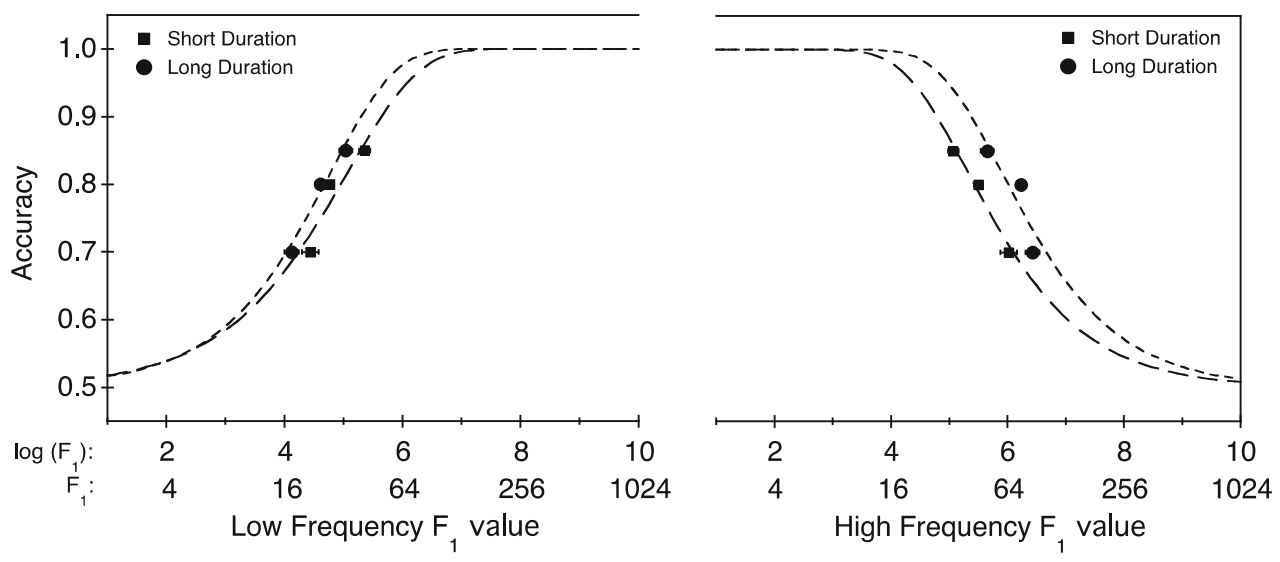

B

DC-C (Description correct—confidence) Condition
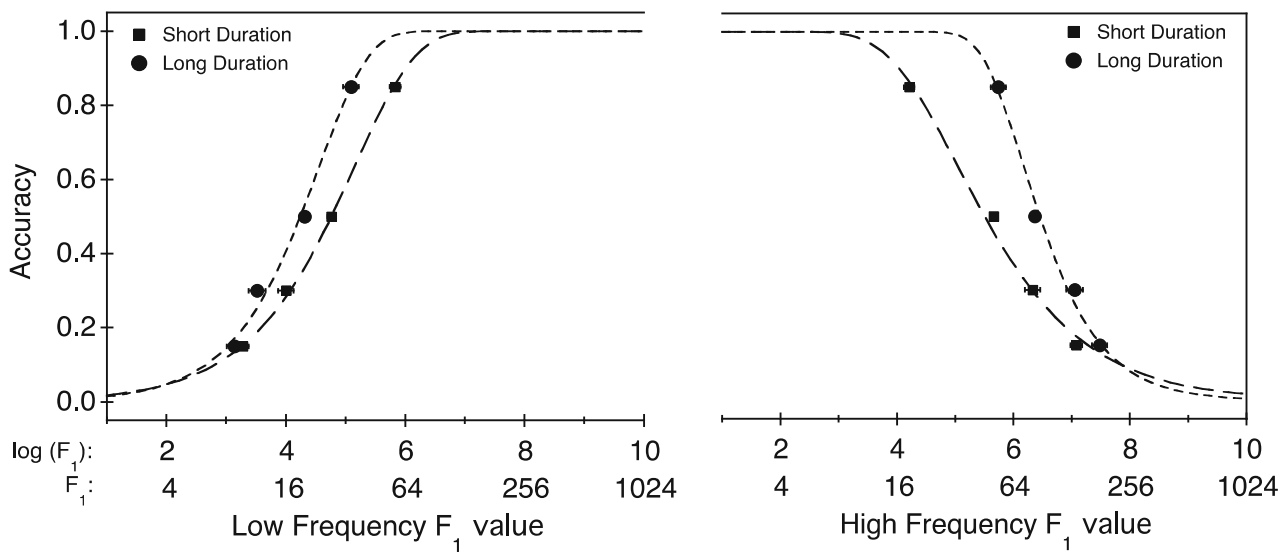

C

UG-YN (Understand gist-yes or no) Condition
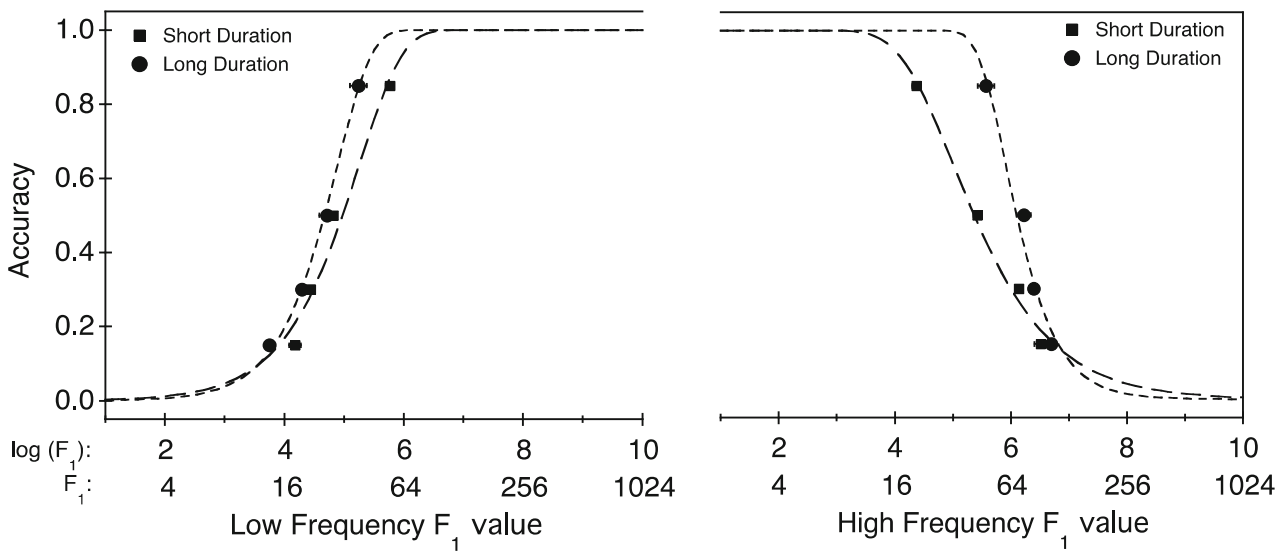

that low-spatial-frequency information was available earlier in time, whereas high-spatial-frequency information needed more time to be processed. More specifically, if low spatial frequencies are mostly analyzed early in time, then there is little space for identification improvement if longer exposure duration is allowed. However, if processing of high spatial frequencies is carried out later, then prolonged exposure duration can (and does) allow better identification. Our results support this scenario, indicating global precedence in the analysis of natural scenes (Kimchi, 1992; Loftus \& Harley, 2004; Navon, 1977; Sanocki, 1993).

The slope of the psychometric function describes the gathering of information from spatial frequencies. In particular, shallow functions indicate that information is 


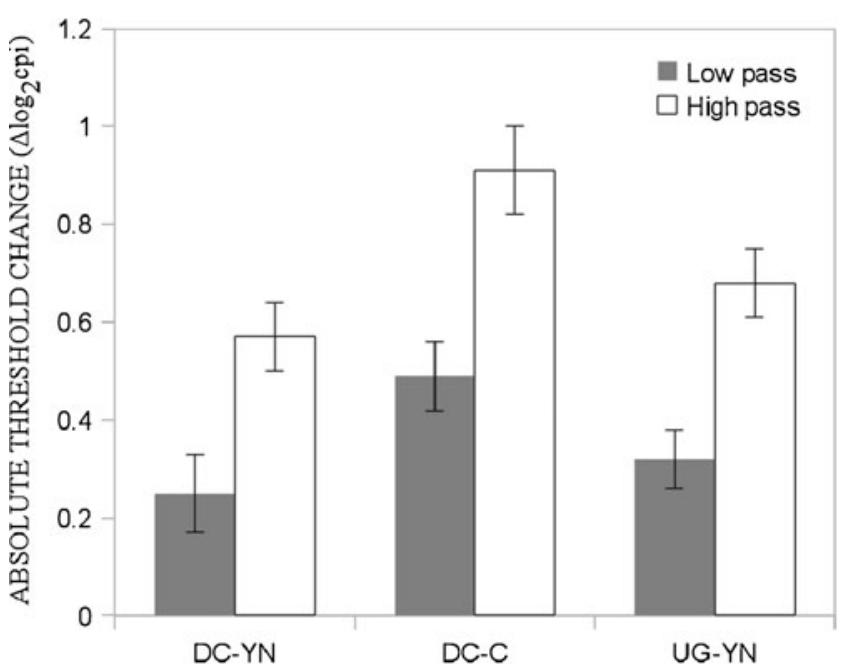

Fig. 3 Absolute threshold change (long exposure - short exposure) for high- and low-passed images, in all task conditions. Error bars represent SEMs

spread across a relatively wide range of frequencies, whereas steep functions indicate that a restricted set of frequencies is sufficient for scene identification (Patterson, Foster, \& Heron, 1980; Strasburger, 2001). That is, shallow functions indicate that little information is gained from spatial frequency information (less efficiency), whereas steep functions are associated with greater identification increases as a function of spatial frequency information (more efficiency). In UG-YN, the psychometric function slope was more affected by exposure duration in the highthan in the low-spatial-frequency range; in particular, steeper (more efficient) functions were observed for longer than for shorter exposure duration. Also, in both UG-YN and DC-C, psychometric functions were steeper for low spatial frequencies than for high spatial frequencies at brief exposure durations, whereas the opposite pattern was observed at longer exposure durations, providing further support to the idea that processing of low spatial frequencies temporally precedes processing of higher spatial frequencies.

Table 1 Thresholds of the psychometric functions ( $\log _{2}$ cpi)

\begin{tabular}{llllll}
\hline & & Short Duration & SEM & Long Duration & SEM \\
\hline DC-YN & High pass & 5.43 & 0.07 & 6.02 & 0.04 \\
& Low pass & 4.95 & 0.06 & 4.68 & 0.09 \\
DC-C & High pass & 5.39 & 0.15 & 6.38 & 0.08 \\
& Low pass & 4.87 & 0.06 & 4.33 & 0.07 \\
\multirow{2}{*}{ UG-YN } & High pass & 5.28 & 0.1 & 6.05 & 0.05 \\
& Low pass & 5.04 & 0.07 & 4.72 & 0.05 \\
\hline
\end{tabular}

Table 2 Slopes of the psychometric functions ( $\left.\log _{2} \mathrm{cpi}\right)$

\begin{tabular}{llllll}
\hline & & Short Duration & SEM & Long Duration & SEM \\
\hline DC-YN & High pass & -1.27 & 0.30 & -1.30 & 0.24 \\
& Low pass & 1.21 & 0.22 & 1.31 & 0.30 \\
DC-C & High pass & -1.18 & 0.20 & -1.98 & 0.41 \\
& Low pass & 1.42 & 0.11 & 1.74 & 0.22 \\
UG-YN & High pass & -1.52 & 0.14 & -2.93 & 0.48 \\
& Low pass & 1.98 & 0.24 & 2.48 & 0.36 \\
\hline
\end{tabular}

Taken together, the present data supplement previous results investigating the temporal order of analysis of spatial frequency information, demonstrating that the use of spatial frequencies proceeds from low to high in face, digit, and scene identification (Hughes, Nozawa, \& Kitterle, 1996; LaGasse, 1993; Loftus \& Harley, 2004; Sanocki, 1993). Converging evidence from neural models of visual processing (e.g., Bar, 2004; Bullier, 2001; Fabre-Thorpe, 2003) also suggests that low and high spatial frequencies are interactively processed, with low spatial frequencies exerting a priming effect on the subsequent processing of fine-grained high spatial frequencies, eventually maximizing identification efficiency.

The present results focus on the role of spatial frequencies in understanding the gist of natural, nonhybrid scenes. However, the visual system is continuously engaged in a variety of tasks (categorization, visual search, etc.), and it has recently been suggested that the use of spatial frequencies may vary depending on the task being carried out (flexible usage hypothesis; Morrison \& Schyns, 2001). Therefore, a natural continuation of this work would be to generalize the present results to other tasks, focusing on those that presumably rely more on local than on global features.

Image statistics and information gathering

In all three tasks, psychometric functions for high- and lowpassed pictures were remarkably symmetric. This result suggests a link between the statistical properties of natural images and the way the visual system processes the visual input. In particular, previous results have shown that the integrated contrast energy over a frequency range depends only on the log width of the frequency range itself, and is hence constant for equal-ratio frequency ranges. For instance, integrated contrast energy in the $4-8$ cycles/degree (cpd) range is approximately equal to that in the 16-32 cpd range (Field 1987; Hughes et al., 1996). This interesting statistical property of natural images provides a basis for scale invariance, since the integrated contrast energy of a frequency band does not vary when a stimulus is moved 
away from the observer and its retinal spatial frequency increases (Field, 1987).

Several results suggest that this statistical property of visual images may be represented in the functioning of the visual system (Hughes et al., 1996; Van Hooser, 2007). In particular, to support the integration of contrast changes over frequency intervals that are spaced on a ratio scale, one possibility is that the visual system analyzes the incoming input according to spatial frequency channels whose width is proportional to the central frequency. This possibility is consistent with the observation that in humans, as well as in several mammals, the bandwidth of spatial frequency channels is best described by a ratio scale and is approximately constant for high and low spatial frequencies (Stromeyer \& Klein, 1974; Van Hooser, 2007).

The present data are in good agreement with the possibility of constant ratio bandwidth across spatial frequencies. More specifically, since integrated contrast energy is approximately constant across the spatial frequency spectrum, the hypothesis that spatial frequency channels are distributed on a ratio scale predicts that the amount of information gathered from a filtered version of a picture is proportional to the width of the frequency range examined, but not to its type (low or high pass). This is exactly what was observed, and psychometric functions for low- and high-passed pictures were symmetric on a logarithmic scale.

Finally, in the present study, psychometric functions exceeded $80 \%$ accuracy $(55 \%$ in DC-YN, when guessing was allowed) in a range of approximately 16 to $90 \mathrm{cpi}$. This result suggests that the availability of these intermediate frequencies, embedded in an either low-passed or highpassed picture, may allow for the identification of visual scenes (Gold, Bennett, \& Sekuler, 1999; Parish \& Sperling, 1991). Converging evidence supporting this possibility comes from the finding that, in the primate visual cortex, most cells are tuned to intermediate spatial frequencies, whereas fewer cells are selective for extremely low or high spatial frequencies (Silverman, Grosof, De Valois, \& Elfar, 1989).

\section{Conclusion}

The results of the present study indicated that, on the basis of the different types of information conveyed by low and high spatial frequencies, participants could confidently and accurately identify natural scenes following either highpassed or low-passed versions of the same pictures, with very similar psychometric functions. Supporting the notion of global precedence in the identification of natural scenes, processing of high spatial frequencies was more hindered than that of low spatial frequencies when temporal availability of a visual scene was reduced.

Author Note This study was partially supported by NIMH Grant MH41637 to G. R. Loftus. The authors thank Maurizio Codispoti and John Palmer for helpful discussion, and Bill Peria and Becky Nichols for their support in conducting the study.

\section{References}

Bar, M. (2004). Visual objects in context. Nature Reviews Neuroscience, $5,617-629$.

Bullier, J. (2001). Integrated model of visual processing. Brain Research Reviews, 36, 96-107.

De Cesarei, A., \& Codispoti, M. (2008). Fuzzy picture processing: Effects of size reduction and blurring on emotional processing. Emotion, 8, 352-363.

De Cesarei, A., \& Codispoti, M. (2010). Effects of picture size reduction and blurring on emotional engagement. PloS One, 5 (10), e13399.

Fabre-Thorpe, M. (2003). Visual categorization: Accessing abstraction in non human primates. Philosophical Transactions by the Royal Society B, 358, 1215-1223.

Field, D. J. (1987). Relations between the statistics of natural images and the response properties of cortical cells. Journal of the Optical Society of America A: Optics and image science, 4, 2379-2394.

Gold, J., Bennett, P. J., \& Sekuler, A. B. (1999). Identification of bandpass filtered letters and faces by human and ideal observers. Vision Research, 39, 3537-3560.

Hughes, H. C., Nozawa, G., \& Kitterle, F. L. (1996). Global precedence, spatial frequency channels, and the statistic of the natural image. Journal of Cognitive Neuroscience, 8, 197-230.

Kimchi, R. (1992). Primacy of wholistic processing and global/local paradigm: A critical review. Psychological Bulletin, 112, 24-38.

LaGasse, L. L. (1993). Effects of good form and spatial frequency on global precedence. Perception \& Psychophysics, 53, 89-105.

Levitt, H. (1971). Transformed up-down methods in psychoacoustics. Journal of the Acoustical Society of America, 49, 467-477.

Loftus, G. R., \& Harley, E. M. (2004). How different spatial-frequency components contribute to visual information acquisition. Journal of Experimental Psychology. Human Perception and Performance, 30, 104-118.

Loftus, G. R., \& Harley, E. M. (2005). Why is it easier to identify someone close than far away? Psychonomic Bulletin \& Review, $12,43-65$.

Morrison, D. J., \& Schyns, P. G. (2001). Usage of spatial scales for the categorization of faces, objects, and scenes. Psychonomic Bulletin and Review, 8, 454-469.

Navon, D. (1977). Forest before trees: The precedence of global features in visual perception. Cognitive Psychology, 9, 353383.

Parish, D. H., \& Sperling, G. (1991). Object spatial frequencies, retinal spatial frequencies, noise, and the efficiency of letter discrimination. Vision Research, 31, 1399-1415.

Patterson, V. H., Foster, D. H., \& Heron, J. R. (1980). Variability of visual threshold in multiple sclerosis. Effect of background luminance on frequency of seeing. Brain, 103, 139-147.

Pelli, D. G. (1987). On the relation between summation and facilitation. Vision Research, 27, 119-123.

Pelli, D. G., \& Zhang, L. (1991). Accurate control of contrast on microcomputer displays. Vision Research, 31, 1337-1350. 
Sanocki, T. (1993). Time course of object identification: Evidence for a global-to-local contingency. Journal of Experimental Psychology. Human Perception and Performance, 19, 878-898.

Schyns, P. G., \& Oliva, A. (1994). From blobs to boundary edges: Evidence for time and spatial scale dependent scene recognition. Psychological Science, 5, 195-200.

Shulman, G. L., \& Wilson, J. (1987). Spatial frequency and selective attention to local and global information. Perception, 16, 89-101.

Silverman, M. S., Grosof, D. H., De Valois, R. L., \& Elfar, S. D. (1989). Spatial-frequency organization in primate striate cortex. Proceedings of the National Academy of Sciences, 86, 711-715.
Strasburger, H. (2001). Converting between measures of slope of the psychometric function. Perception \& Psychophysics, 63, $1348-1355$.

Stromeyer, C. F., \& Klein, S. (1974). Spatial frequency channels in human vision as asymmetric (edge) mechanisms. Vision Research, 14, 1409-1420.

Ulrich, R., \& Miller, J. (2001). Using the jackknife-based scoring method for measuring LRP onset effects in factorial design. Psychophysiology, 38, 816-827.

Van Hooser, S. D. (2007). Similarity and diversity in visual cortex: Is there a unifying theory of cortical computation? The Neuroscientist, 13, 639-656. 